\title{
The next generation IUD introduced in Turkey
}

The intrauterine device (IUD) is the main method of contraception worldwide, including Turkey, according to a cross-sectional study among 6,506 women in 15-49 age group study conducted by Pala et al published in 2008 (1). This study also reported a high incidence of anemia in copper IUD users.

Conventional framed T-shaped devices (TCu380A, Mirena; Bayer HealthCare Pharmaceuticals Inc.; NJ, USA), given their size limitations (32 $\mathrm{mm}$ width), whether used in parous or nulliparous women, have several disadvantages as they have to fit the uterine cavity of all women regardless of shape or size in order to be well tolerated. However, a growing body of evidence exists confirming that the uterine cavity size varies significantly amongst women and most devices of singular dimensions do not fit all uterine cavities. Studies of the uterine cavity show that uterine cavities differ from woman to woman ranging from $<10 \mathrm{~mm}$ to $>40 \mathrm{~mm}$. Discrepancy between the IUD and the uterine cavity can often result in partial or total expulsion due to severe uterine contractions. If not expelled, pain, cramping and abnormal bleeding are common complaints caused by malposition, displacement or embedment of the IUD. Malposition and displacement of an IUD, resulting in embedment and/or perforation are being reported more frequently as a consequence of increasing use of 3D sonographic or hysteroscopic methods in the routine clinical setting. Recently, a frameless copper IUD (GyneFix ${ }^{\circledR}$ ) was introduced in Turkey. The IUD consists of a number of copper cylinders attached to a surgical nonabsorbable polypropylene suture thread with a preformed surgical knot on top which, upon contact with the fundus is inserted into the fundus of the uterus. The depth of the insertion is controlled by the specially designed inserter (Figure 1). As the IUD is anchored in the fundus, it cannot be expelled as a consequence of uterine contractions, which can at times be quite severe especially during menstruation. Removal has been found to be easy and with minimal patient discomfort. Return to fertility as with most copper IUDs is almost immediate. Clinical trials conducted in Germany and other regions in Europe have demonstrated a high acceptance and continuation rates by women. Furthermore, due to its small size and lack of transverse arms, the amount of menstrual bleeding that occurs, unlike that seen with framed copper devices such as TCu380A, remains unchanged. The device is only $2 \mathrm{~cm}$ long and $2.2 \mathrm{~mm}$ in width and uses thick-walled copper cylinders capable of releasing copper from both the internal and external surfaces. As the frameless IUD design lacks a plastic frame and utilizes multiple individual copper cylinders it is completely flexible in utero, resulting in high tolerance and continuations rates reported in clinical studies ( $>90 \%$ over 5 years). By comparison the continuation rates for TCu380A, published in the literature,

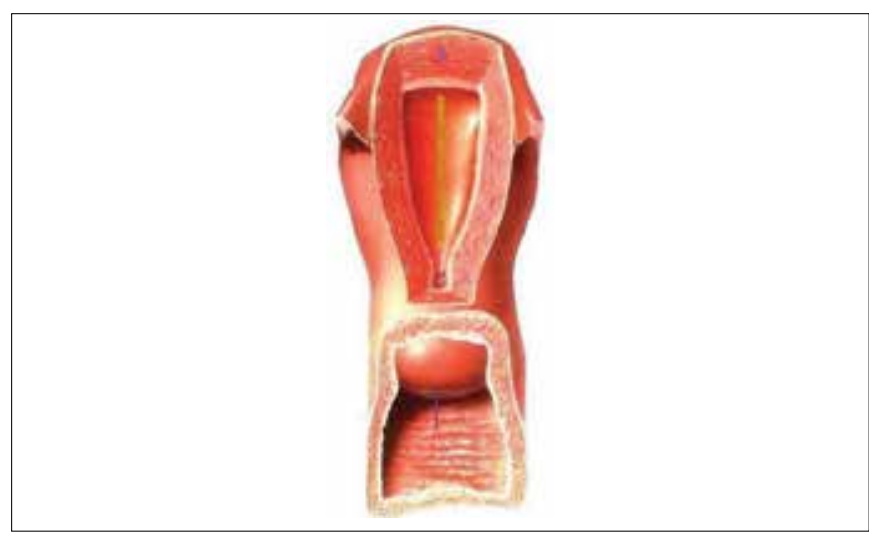

Figure 1. Illustration of the frameless IUD in a uterine cavity

are in the order of 40 to $50 \%$ at 5 years. In addition, multicenter efficacy studies confirm equal or slightly higher efficacy compared with the TCu380A IUD in randomized clinical trials (2). One consequence of the anchored design is that it affords release of copper high up in the uterine cavity in the vicinity of the tubal ostia which serves to maximize effectiveness and limit ectopic pregnancies to almost zero, as confirmed in large clinical trials. The lifespan of the different device types can vary between 3 and 10 years. These devices are CE-marked and some of them are currently available for use in Turkey.

Given its novel anchoring and insertion methodology, and the lack of familiarity of most physicians and surgeons with the intrauterine implant (IUI), the insertion technique requires some degree of formalized training. Doctors, even experienced with the conventional IUDs, need to become familiar with the insertion technique. Proper positioning of the anchor in the uterine fundus is required to guarantee optimal performance. But, as the technique is straightforward, proficiency is easily acquired, as I experienced myself. An ultrasound check following insertion shows of the anchor has been properly placed in the fundal myometrium.

Assoc. Prof. Gazi Yıldırım, M.D.

Department of Obstetrics and Gynecology, Yeditepe University School of Medicine, Istanbul, Turkey

\section{References}

1. Pala K, Dundar N. Prevalence \& risk factors of anaemia among women of reproductive age in Bursa, Turkey. Indian J Med Res 2008; 128: 282-6.

2. Wildemeersch D, Jandi S, Pett A, Nolte K, Hasskamp T, Vrijens M. Use of frameless intrauterine devices and systems in young nulliparous and adolescent women: results of a multicenter study. Int J Womens Health 2014; 6: 727-34. [Crossref] 